\title{
LA REFUTACIÓN EN LA DISPOSICIÓN MUSICAL BARROCA \\ O CÓMO ARGUMENTAR CON SONIDOS
}

\author{
Gabriel Pérsico \\ Universidad Nacional de las Artes - Argentina \\ Email: gabrielpersico@gmail.com
}

\section{Resumen:}

Estudiaremos en este trabajo algunos de los distintos recursos de oposición argumental, basados en la retórica textual, que se emplearon en la música barroca. Ejemplificaremos con fragmentos del Aria Seele, para soprano y flauto traverso obligato, del Oratorio de Pascua, BWV 249 de Johann Sebastian Bach.

Palabras clave: Argumentación. Confutatio (refutación). Retórica musical. Música barroca

Rebbutals in musical disposition, or how to argument with sounds.

\section{Abstract:}

This paper is focused on several argumentative resources based on textual rhetoric and employed in baroque music. Excerpts of Aria Seele, for soprano and flauto traverso obligato from Johann Sebastian Bach's Eastern Oratorium BWV 249 will be analysed.

Keywords: Argument. Confutatio (rebuttal). Musical Rhetoric. Baroque music. 
1. El diccionario latino VOX, define la entrada contentio con los siguientes significados: esfuerzo, elevación (de la voz, del tono), pasión, ardor, celo; lucha; debate, discurso vehemente, apasionado; comparación; antítesis. Queda claro entonces el carácter inestable y confrontativo de la sección en donde se enfrentan las pruebas contrarias en el discurso forense y donde se desarrollan los motivos y/o se oponen los afectos en el discurso musical.
La metáfora de la música como lenguaje, de larga data ya a principios del siglo XVIII, concebía a la música como un discurso sonoro. Los teóricos desarrollaban herramientas de análisisy composición a partir de la retórica textual. La forma podía ser asimilada a la disposición de un discurso, en especial a un discurso del género forense. Johann Mattheson en sus escritos aborda el problema de la disposición aplicada a la música. Otros autores contemporáneos también hacen referencia al asunto en mayor o menor grado. Consideramos que la clara percepción de la disposición musical es fundamental para un intérprete historicista. En ella, la identificación de los mecanismos musicales de refutación resulta de fundamental importancia y configura un primer paso para la conciencia del despliegue del discurso completo.

\section{ARGUMENTATIO (CONTENTIO): PROPOSITIO, CONFIRMATIO, CONFUTATIO, DIVISIO}

Quintiliano sostiene que lo que define al género forense es la oposición entre la acusación y la defensa. Dice además que resulta más fácil acusar que defender. Como el defensor deberá negar y refutar las pruebas que se le presentan, deberá poseer mayor elocuencia. Por esa razón, las secciones que configuran estas funciones no están dogmáticamente definidas y variarán según el tipo de discurso que se precise elaborar.

Los autores clásicos denominan argumentación, también llamada contentio ${ }^{1}$, a la parte del discurso que agrupa las secciones de confutatio y refutatio. Entendida como el meollo del discurso, es la sección que sirve para establecer la credibilidad del punto de vista que defendemos. Es el momento en que se produce el agonismo dialéctico entre el argumento sostenido y su contrario. Estas dos secciones principales presentan entonces una función específica:

1) la Confutatio o Refutatio que consiste en una argumentación negativa que intenta demostrar lo insostenible de la posición contraria y sus pruebas.

2) la Confirmatio o Probatio que consiste en una argumentación positiva que demuestra la credibilidad de nuestra causa y la validez de sus pruebas. 
La proposición (propositio) previa tiene la función de presentar el verdadero contenido y propósito del discurso. La tradicional división en principium, medium, finis es complejizada por los teóricos alemanes al utilizarla en relación a la fuga con el esquema de la chria lógica. El esquema estructural de la chria es una variante pedagógica del esquema de disposición clásica de la retórica. Butler cita la comparación de ambos esquemas de Christoph Weissenborn ${ }^{2}$, uno de los más importantes retóricos alemanes del período:

\begin{tabular}{|c|c|c|}
\hline \multicolumn{2}{|l|}{ CHRIA } & DISPOSITIO \\
\hline \multicolumn{2}{|l|}{$\ldots \ldots .}$. & exordium \\
\hline \multicolumn{2}{|l|}{$\ldots \ldots}$. & narratio \\
\hline \multicolumn{2}{|l|}{ prótasis } & propositio \\
\hline \multicolumn{2}{|c|}{ aetiologia (probatio) } & ....... \\
\hline \multicolumn{2}{|c|}{........ } & divisio \\
\hline \multirow[t]{4}{*}{ amplificatio } & - a contrario (per confutationem) & \multirow{4}{*}{$\begin{array}{l}\text { confutatio (secciones agrupadas bajo el término } \\
\text { contentio, lo que señala su reversibilidad; pruebas y } \\
\text { argumentos para reforzar o refutar el tópico } \\
\text { principal) }\end{array}$} \\
\hline & - a comparato & \\
\hline & -ab exemplo & \\
\hline & -a testimonio & \\
\hline \multicolumn{2}{|l|}{........ } & confirmatio \\
\hline \multicolumn{2}{|c|}{$\begin{array}{l}\text { conclusio (o repetición de prótasis, o } \\
\text { consecuencia lógica en forma de axioma o } \\
\text { deseo, comúnmente en forma de un verso corto) }\end{array}$} & conclusio \\
\hline
\end{tabular}

La chria carece de las dos secciones iniciales de la dispositio clásica, comienza "in media res" con la prótasis o tesis principal, sinónimo de propositio. En la chria, entonces,la narración no aparece, ya que podía estar subsumida en la sección de proposición. Para Quintiliano, la narración contaba los hechos y la proposición se focalizaba en las pruebas que debían ser defendidas. Por eso es necesario que la proposición siga a la narración: una vez que los hechos fueron expuestos se procede a presentar las pruebas. Por ello también, la proposición está antes de la confirmación que constituye una de las secciones de la contentio o argumentatio:

La narración, según el orden natural, precede a la confirmación, pues debemos probar lo que primero hemos contado para este fin $[\ldots]$

Dicen algunos que la proposición, como parte de la causa judicial, debe seguir a la narración. A cuya opinión respondo diciendo que por proposición entiendo el principio de toda confirmación. Ésta no solamente se pone antes de las pruebas, sino algunas veces al principio de cada una de ellas [...](QUINTILIANO, 2004, cap. IV).
2. Grüindliche Einleitung zur Teutschen und

Lateinischen Oratorie und Poesie (Dresden y Leipzig, 1731), p. 93-94. En (BUTLER, 1977, p. 70) 
3. Lenneberg traduce aquí "la proposición misma": the proposition itself. (LENNEBERG, 1958, p. 194).

\section{Harrys en su} traducción de del Capellmeister, invierte el orden de los parágrafos 10 y 11, probablemente para que coincidan con la lista del parágrafo 4 .

5. "Las siguientes dos secciones en el esquema clásico de disposición, confirmatio y confutatio, envuelven ambas la declaración de argumentos, la primera para reforzar el caso, la última para refutar los argumentos presentados contra él por la parte contraria. No sorprende entonces que estas dos secciones aparezcan a menudo unificadas bajo el término común de contentio, y su secuencia particular en el esquema de la dispositio parezca reversible." ["The next two sections in the classical disposition scheme, confirmatio and confutatio, both involve the statement of arguments, the former to strengthen the case, the latter to refute arguments brought against it by an opposing party. Thus it is not surprising that these two sections were often linked together under the single term contentio, and their particular sequence in the dispositio scheme seems to be reversible"] (BUTLER, 1977, p. 70).
Mattheson define la propositio de la siguiente manera:

La Propositio, o el discurso propiamente dicho ${ }^{3}$, contiene brevemente el contenido [sentido] o finalidad [propósito] del discurso musical, y es de dos tipos: simple o compuesto, a la cual también pertenece la Propositio variada u ornamentada en música, que no es mencionada en la retórica [textual] [...] (Mattheson \& Harris, 1739, p 471) (LENNEBERG, 1958, p. 194-195).Nẫo entendí esta citaçẵo!

A continuación en el mismo parágrafo, Mattheson anticipa brevemente su análisis posterior de las proposiciones (simple, variada y compuesta) en el aria de Marcello. Recordemos que el autor ejemplifica su análisis de la disposito en un aria da capo, sorprendentemente, sin proporcionar el texto. Por lo que se deduce en su análisis, las diferentes proposiciones se identifican con la aparición en diversas formas del tema o motivos principales del aria en la sección A de la misma. Este funcionamiento se relaciona de alguna manera con el recurso del motto en las arias italianas de los siglos XVII y XVIII.

En los parágrafos siguientes $(\$ 10$ y $\$ 11)$, Mattheson define la Confirmatio y la Confutatio. Es notorio que el orden de los parágrafos esté invertido con respecto a la lista de secciones de la dispositio que figura en el $\$ 4$. Ahora aparece en primer lugar la confutación y luego la confirmación ${ }^{4}$. En este orden aparecerán luego en el análisis del aria, ya que las confutaciones forman parte de la sección B de la misma y se considerará confirmación la vuelta a la sección A. Para Bartel, ambos secciones son habitualmente agrupadas bajo el término de contentio (ver ut supra) y su ubicación en la dispositio pareciera ser reversible, lo cual justificaría su diferente ordenamiento en el capítulo de Mattheson que estamos estudiando. (BARTEL, 1997, p. 81). Butler sostiene lo mismo ${ }^{5}$, explicando que Weissenborg en su esquema de la chria, parece dejar claro que los diferentes métodos de amplificatio son equivalentes a la confutatio: 
[...] Esto se confirma por la aparición de la confirmatio siguiendo a los diferentes métodos de amplificación y justo antes de la conclusio en la adaptación de la chria a la fuga de Schmidt(BUTLER, 1977: n. 91, p. 105)'.

Esta reversibilidad representa una posible aplicación flexible del esquema retórico de la dispositio a la música. Además, podemos constatar en los análisis que la sección de contentio o argumentatio muchas veces incluye más de un segmento con funciones de confutatio o confirmatio.

Veamos entonces cómo Mattheson define estas secciones:

$\$ 10$ La Confutatio es la resolución de las objeciones. En música esto puede ser expresado por medio de retardos ${ }^{7}$ o por citar y refutar pasajes aparentemente extraños. Estos contrastes, cuidadosamente empleados, son una especial fuente de placer auditivo. Todo lo que vaya en contra de la proposición se resuelve y acomoda. Esta parte de la disposición, a pesar de ser una de las más bellas, no se encuentra a menudo en la música." (LENNEBERG, 1958, p. 195) .

\$11 La Confirmatio es el ingenioso refuerzo de la proposición y aparece en melodías por medio de repeticiones sorprendentes (que no hay que confundir con las "reprises" comunes"). Lo que tenemos en mente son pasajes placenteros repetidos varias veces con todo tipo de variaciones agradables [...] (LENNEBERG, 1958, p. 195) ${ }^{10}$.

Es claro en sus definiciones el carácter positivo de la confirmatio, en el sentido de corresponder motívicamente a lo planteado en la proposición y probablemente en el exordio también. La repetición de pasajes que refuerzan la proposición, variándolos, parecen suponer que se produjo previamente una oposición a los mismos. Mattheson pone el énfasis en la variación motívica, al igual que los tratadistas que abordan retóricamente a la fuga lo hacen con respecto a las variaciones que el sujeto de la misma puede sufrir. No hay aquí demasiado acento en las variaciones tonales, que para el pensamiento del autor, están íntimamente ligadas a la expresión de los afectos. Sin embargo, más adelante en el mismo capítulo al analizar la parte B del aria da capo hay referencia la diferencia semántica que produce en el mismo motivo el cambio de centro tonal.
6. "This is confirmed by the appearance of the confirmatio following the various methods of amplification and just prior to the conclusio in Schmidt's adaptation of the chria to fugue".

7. Encontramos aquí una discrepancia en las traducciones inglesas. Lenneber traduce por "ties" y evidentemente duda ya que coloca entre corchetes el término alemán [Bindung]. Harrys traduce "combining", utilizando otra estructura de oración. Lenneberg. pareciera referirse entonces a "retardos", una de las posibles traducciones técnicas de ties, mientras que Harrys haría referencia a elaboraciones motívicas. Ambas traducciones tienen sentido. Me inclino por la primera ya que hace alusión a la aparición de disonancias, mientras que la elaboración motívica está implícita en el final de la oración de Mattheson.

8. "Confutatio is the resolution of objection. In music it may be expressed by means of ties [Bindungen] or by the citation and refutation of apparently foreign passages. Such contrasts, carefully used, are a special source of aural pleasure. Everything that goes against the proposition is resolved and settled. This part of disposition, al-though it is one of the most beautiful, is not found in music very often".

9. Lenneberg aclara que Mattheson con "reprises" se refiere a las repeticiones de secciones enteras, indicadas con un signo.

10. "Confirmatio is the clever reinforcement of 
the proposition and is brought about in melodies by means of surprising repetitions (by which we do not mean the ordinary reprises). What we have in mind are agreeable passages repeated several times with all sorts of nice variations".

11. "Nellealtre Parti, non potendonemenohavere i Soggettiinteri, si addatteranno i
La confutación entonces pareciera que produce la oposición al asunto principal de la música no sólo por cambios motívicos sino también por la aparición de disonancias y modulaciones. En el análisis del inicio de la parte B del aria(Cap. XIV, \$20), Mattheson indica una serie de cambios de centro tonal y luego de un pasaje con retardos, la llegada a la tónica de re menor (cuarto grado) a través de una cadencia con la siguiente sentencia "confutatio, rhetoribusdissolutio, nobisresolutio". O sea: "Confutación, para los rétores disolución, para nosotros [los músicos] resolución”. No queda claro por qué el autor sostiene que pese a ser una de las más bellas partes del discurso, raramente se encuentra en la música. Posiblemente, podemos aventurar, esto se origine en la tradición alemana de análisis retórico-musical del repertorio imitativo y de la fuga, en donde la confutatio se realiza a partir de elaboraciones del sujeto y contrasujeto y de recursos contrapuntísticos. Sin embargo, en el repertorio que Mattheson denomina "moderno", el más relacionado con la ópera italiana, los recursos de oposición son más amplios.

Al tratar la sección de confutatio en su análisis retórico de la fuga, Butler señala que la fragmentación temática es un recurso muy utilizado y teorizado por los alemanes.

Ya Mattheson en su capítulo sobre la invención melódica (Cf. capítulo IV, $\$ 15$ y ss.) del Capellmeister partía de la fragmentación para la creación de un nuevo sujeto.

Butler también cita al teórico italiano Giovanni Bontempi (c. 1624-1705) que ejemplifica la elaboración motívica que puede ocurrir en la confutación de una fuga a partir de unidades menores del sujeto que denomina "particelle".

En las otras partes, no pudiendo tampoco tener los Sujetos completos, se adaptarán sus miembros, que son las "particelle" [partecitas] de los mismos Sujetos [...] $]^{11}$ (apud BUTLER, 1977, p. 80).

El proceso de la fragmentación de los motivos es habitualmente designado en la teoría retórico-musical como distributio, la cual en realidad es una figura o tropo que a veces también es designada con el término de divisio. El término puede nombrar a una sección de la disposición del discurso y algunos autores así la tratan. Su importancia radica en la elaboración temática de raíz lógica, ya que construye a partir de unidades menores. Butler también cita al teórico Johann 
Adolph Scheibe (1708-1706) que comenta la figura retórica de distributiode la siguiente manera:

[...] disección (distributio). Aparece cuando se trabaja el principal sujeto de una pieza de tal manera que se observa especialmente cada una de sus partes, una después de la otra. Si se quiere quizás disecar el tema de una fuga que era un poco largo, entonces se debe primero tratar una frase o compás y luego también el fragmento que queda de manera similar, y consecuentemente se podrían considerar todas las partes del sujeto principal como frases especiales y aislar las unas de otras por tratamientos diferentes. Esta figura contribuye de gran manera a la completa e ingeniosa elaboración de una fuga [...]Der critische Musikus (Hamburgo, 1745, p. 692-93), (apud BUTLER, 1977, p. 83) ${ }^{121}$.

Este proceso de elaboración por división es una de las características más importantes de la sección de la confutación, además de la aparición de disonancias o "melodías extrañas" como afirma Mattesohn.

Johann Nikolaus Forkel (1749-1818) es uno de los teóricos que consideran la división como una sección de la dispositio. Afirma que en la fuga se encuentra luego de la primera exposición temática (BUTLER, 1977, p. 84). Lo interesante de su planteo es que caracteriza el procedimiento en relación a la precisa expresión de los afectos, de la misma manera en que lo hace el lenguaje, utilizando sinónimos, paráfrasis, etc:

La subdivisión de un tema principal sirve para examinar el material musical desde varios puntos de vista [...] La intención de una composición es expresar tanto un sentimiento individual como uno general. En ambos casos las relaciones y circunstancias son tan diversas que los sentimientos no pueden ser aclarados suficientemente sin disolverlos en partes separadas. Esta disolución emplea recursos similares a los lingüísticos: por ejemplo, en música tenemos también expresiones sinónimas, diversas formas de paráfrasis, reemplazos [hyperbaton], etc. Incluso una individualización de sentimientos generales puede ser expresada musicalmente [...] ${ }^{13}$ Allgemeine Geschichteder Musik. Leipzig, 17881801(apud BARTEL, 1997, p. 241-242, grifo nuestro).
12. "[...] is dissection (distributio). This occurs when one works out the principal subject of a piece in such a way that one dwells upon each part of it specially, one part after another. If one wished perhaps to dissect the thema of a fugue that was somewhat long, then one would first treat one phrase or measure and then also fragment in a similar manner what remained, and consequently would consider all parts of the principal subject as special phrases and isolate these from one another by different treatment. That this figure contributes a great deal to the skillful and consummate working out of a fugue, $[\ldots]$ ”.

13. "The subdivisions of a principal theme serve to examine the musical material from all sides [...] A composition's intention is to express either an individual or a general sentiment. In both cases the relationships and circumstances are so diverse that the sentiments cannot be sufficiently clarified without dissolving them into their separate parts. Such a dissolution employs devices similar to linguistic ones: for example, in music we also have synonymous expressions, diverse forms of paraphrase, replacements [hyperbaton], and so on. Even an individualization of general sentiments can be musically expressed [...]”. 
Sinteticemos algunos de los procedimientos citados que podemos encontrar en la sección de confutatio con el fin de producir la refutación del asunto propuesto:

- disonancias

- retardos

- modulaciones

- segmentos sintácticos contrastantes ("pasajes extraños" para Mattheson, también apostrophes)

- fragmentación (divisio) y elaboración motívica

- afectos que divergen o contrastan con el afecto principal. Individualización de sentimientos generales (Forkel)

Veamos ahora cómo funcionan estos conceptos con un ejemplo.

\section{LA CONFUTATIO EN EL ARIA SEELE}

Analizaremos brevemente el Aria Seele, deineSpecereiensollennichtmehrMyrrhensein, para soprano con flautotraversoobligato del Oratorio de Pascua (Kommt, eiletundlaufet, ihrflüchtigenFüße), versión de 1748/9, BWV 249 de Johann Sebastian Bach. El objetivo es observar cómo la divisio o fragmentación y alguno de los otros elementos enumerados más arriba son utilizados a fin de oponerse al asunto principal del Aria en la sección de la confutatio. El análisis podría enfocarse sólo en el aspecto motívico sin considerar al texto, de la misma manera en que lo hace Mattheson con el aria de Marcello, pero igualmente haremos alusión a algunos aspectos del texto y el contenido general de la pieza.

Se trata de un aria da capo, con una larga sección A y una sección B más breve. El da capo está indicado completo, por lo que el peso formal de la sección A es considerable. Siguiendo a Mattheson, distinguimos una dispositio completa en la sección A. La sección B (medium, para la dispositio tripartita) cumple el rol de confutatio en el esquema $\mathrm{ABA}$, con una modificación en el afecto principal y una pequeña novedad temática, aunque los motivos siguen siendo fundamentalmente los mismos. En el análisis, nos referiremos al exordio a cargo de la flauta y a la confutatio de la sección A. Haremos una breve alusión a los 
cambios motívicos que se producen en la sección B relacionados con la confutación.

El contenido afectivo del aria y también algunos detalles de los motivos presentan vínculos con el Adagio que configura la sección B (medium) de la Sinfonía inicial de la cantata. Las secciones A y A' (principium, finis) de la Sinfonía se hallan en re mayor y están instrumentadas con un gran despliegue de trompetas y timbales, como corresponde al afecto dominante de una cantata cuyo asunto es la Resurrección ${ }^{14}$. Sorprendentemente, tanto el Adagio como el Aria, ambos en si menor, son de una tristeza profunda y una gran melancolía que no pareciera adecuada al tópico de exultación. Por otra parte, la duración del Aria, con su extensa sección A, es considerablemente mayor con respecto a las otras piezas que conforman la cantata.

Desde el punto de vista del contenido semántico y sintáctico el Aria es de una gran complejidad que por supuesto, no agotaremos aquí. El texto, que pertenece a Christian Friedrich Henrici (Picander), es el siguiente:

\section{SecciónA Seele, deineSpezereien}

Alma, tus aromas

SollennichtmehrMyrhen sein.

ya no serán de mirra.

\section{SecciónB Dennallein}

Porque sólo

MitdemLorbeerkranzeprangen,

conel adorno de la corona de laurel

StilltdeinängstlichesVerlangen.

se aliviará tu angustioso anhelo. ${ }^{15}$

El poema hace referencia al relato de los evangelios en el cual las mujeres van a ungir con mirra el cadáver de Jesús para los rituales funerarios. La mirra es una resina que se disolvía en aceite y que tenía, además de perfume, efectos embalsamadores. Cuando las mujeres arriban a la tumba, la encuentran abierta; el cadáver ha desaparecido. Alguien les dice (después se sabe que es un ángel) que Jesús resucitó. Las mujeres quedan aterrorizadas frente a lo sobrenatural, por lo que podríamos aventurar que los afectos principales son de angustia,
14. La versión original de esta cantata era la Schäferkantate, 'Cantata Pastoral' (Drama per Musica) destinada al cumpleaños del Duque Christian of SachsenWeißenfels, en 1725. Bach adapta la pieza para su uso litúrgico a principios de 1730 y la titula Oratorio. Las partes originales de la versión "Pastoral" se han perdido.

15. Agradecemos a la Prof. Sandra Girón por las sugerencias en la traducción y a la Lic. Clara Cortazar por las implicancias hermenéuticas y litúrgicas del texto. 
desasosiego o estupor. Aquí termina el Evangelio según San Marcos en su versión original.

El alma sube al cielo al igual que los aromas y vapores de la mirra, lo cual parece que no será posible porque el cuerpo no está en la tumba y el rito fúnebre no se pudo consumar.

Es interesante el rol de la flauta, ya que prácticamente no comparte sus motivos con la parte de soprano. Pareciera que el instrumento asume la función de exponer los afectos principales de las mujeres o del alma misma, convirtiéndose en una especie de personaje trágico que mima sus pasiones sin palabras. Este procedimiento dramático de "personificar" un instrumento lo usa Bach, por ejemplo, en el Aria para contralto "Ebarmedich" de la Pasión según San Mateo, en donde la "perfección” de la línea del ritornello del violín nunca es alcanzada por la voz que jamás canta una versión completa del mismo. Sumado al hecho de que el acompañamiento está armado con el "halo" de cuerdas que enmarca a Jesús, podríamos suponer que la línea del violín representa la perfección del alma divina que los humanos pretenden alcanzar pero nunca lo logran.(BUTT, 2010, p. 79 y ss.). En nuestra aria, la soprano, por otra parte asume su rol de narrador/ observador como parece sugerirlo el apóstrofe del poema: ella (¿cada uno de nosotros?) le habla al alma. Por eso, es importante entender el rol retórico que asumen los motivos de la flauta en el extenso exordio.

La metáfora del ascenso de los aromas y del alma, está descripta por Bach con la figura de la hipotiposis en la primera sección (ver sección 1 en el ejemplo 1): ascenso (motivo a1) con intervalos cada vez más amplios en gradatio en los compases 2 y 3 . Ya en el exordio observamos el desarrollo motívico, a través de la figura de paronomasia y el ars combinatoria: el motivo b2 se relaciona con la bordadura en a1 y el motivo b3 modificará su último intervalo para configurar al motivo $\mathrm{c} 1$. La sección 2 pareciera relatar el dolor del alma o de las mujeres al introducir el tópico (ampliamente generalizado en la música barroca) de los sollozos o lamentos (motivo c2). El último de los motivos de "sollozo" (c. 6:1) enlaza con una nueva aparición del motivo a1, generando otra variante que denominamos a'. En la sección 3 del exordio aparece un nuevo 
motivo (d) que es la suma y combinación de otros ya escuchados: $\mathrm{b} 2+\mathrm{a} 2+\mathrm{a} 1+\mathrm{b} 1$. También podemos considerar que el motivo que se extiende del c. 9:2 al 9:3:1:1 es una variación (paronomasia) de a1. Luego de dos compases de catabasis melancólica (c. 9 y c. 10), comienza una gradatio en anábasis, con el mismo motivo d (c. 11 a c. 13) que culmina en el re" (c. 14:1) de la sección 4, también la nota más aguda de la anterior sección 1 , para seguir con una rápida catabasis en tresillos (motivo e) que recuerda por una lado al motivo descendente b1 y por otro lado, considerando las dos primeras notas de cada tresillo y luego la bordadura que produce la tercera nota, resulta una variación del motivo de los "sollozos" (c2).

Inmediatamente antes del comienzo del aria, en el final del recitativo, los solistas dicen:

$\begin{array}{cl}\text { Bajo[relator]: } & \begin{array}{c}\text { "con saladas lágrimas } \\ \text { y melancólica añoranza, } \\ \text { le habían destinado una unción, }\end{array} \\ \text { Soprano y alto[mujeres en 1a persona]: } \quad \text { que en vano hemos preparado."16 }\end{array}$

El oyente entonces es inducido a pensar que el exordio de la flauta ilustra el estado de ánimo de las mujeres que encuentran la tumba vacía. Recién en la narratio, la soprano introduce el apóstrofe del texto al alma, lo cual complejiza la situación psicológica: ¡cada una de las mujeres le habla al alma de Jesús?, ¿la soprano es un narrador objetivo apostrofando al alma?, ¿somos cada uno de nosotros dirigiéndonos a nuestra alma o al alma de Jesús? De cualquier manera, queda claro que los diseños motívicos de la flauta y los de la soprano no son los mismos.

Hay otros elementos en el bajo continuo (que no incluimos en el ejemplo) que refuerzan el carácter melancólico del aria como ser la cadencia frigia al final de la primera frase (c. $3: 3$ a c. $4: 1$ ) y el despojado bajo en pizzicato apoyando a la melodía al principio sólo por compás, luego por tiempos (cortos, indicados con corchea y silencio de corchea) en el momento de señalar el tetracordio melancólico que desemboca en la cadencia frigia (c. 3 y c. 4), o avanzando por solemnes yambos.
16. "HatmitgesalznenTränen/Und wehmutsvollem Sehnen/Ihm eine Salbung zugedacht,/Die ihr, wiewir, umsonstgemacht". 


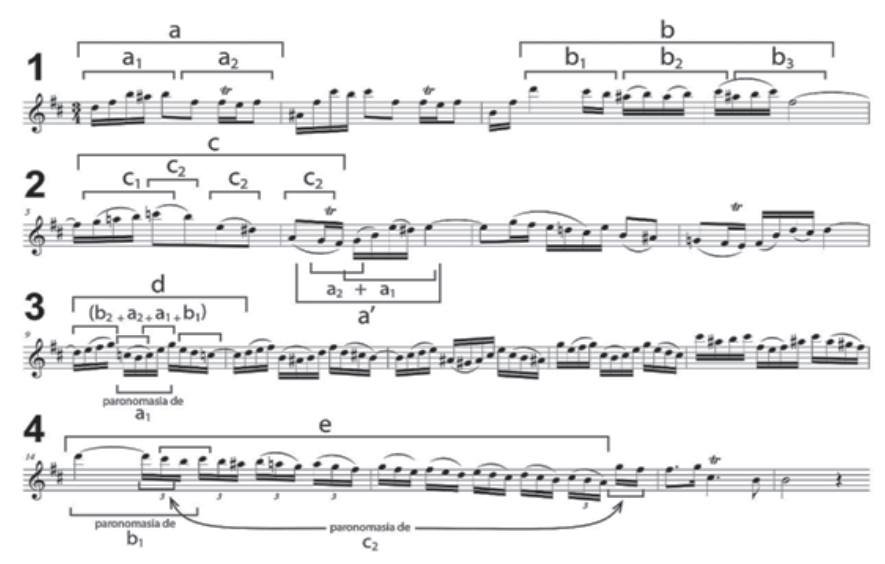

Ejemplo 1: Exordio del Aria Seele, Oratorio de Pascua, BWV 249 de Johann Sebastian Bach

Están claros los procesos de elaboración de motivos que utiliza Bach en el exordio. Coinciden con los que describen Mattheson, Sheibe y Forkel citados más arriba, en especial los relacionados con la fragmentación que da lugar a nuevas configuraciones (figuras de divisio, distributio, paronomasia, etc.). Es interesante hacer notar el contenido afectivo que identifica a los distintos motivos o conjunto de motivos, ya que veremos, de acuerdo con Forkel, el rol que asumirán en la confutación.

Analicemos ahora cómo se produce la confutación de la sección A del Aria (ejemplo 2) y luego nos referiremos brevemente a la sección $\mathrm{B}$ de la misma.

El texto es el mismo que aparece en toda la sección A (ver ut supra), por lo que los recursos refutatorios no están relacionados con el sentido de las palabras. La sección comienza con el motivo principal de la soprano en fa\# menor. Recordemos que Mattheson afirma que si bien los motivos pueden ser idénticos, su significación y función formal pueden cambiar si están transportados a otra tonalidad. De todas maneras, la frase de la soprano no es idéntica ya que presenta un cambio de dirección abrupto con un salto de sexta ascendente al mismo tiempo que comienza la flauta. Si bien no nos vamos a detener en el análisis de los motivos de la voz de soprano, constatamos hasta ahora algunos aspectos propios de la refutación: la tonalidad y la exclamatio o ecphonesis representada por el salto de sexta y el final agudo de la frase ("pasajes extraños" según Mattheson). Constatamos que los motivos de la soprano son diferentes a los de la flauta (salvo quizás por los melismas de tresillos de 
los cc. 51 y 52, pero aquí en la voz son ascendentes, lo cual refuerza el carácter enfático y no depresivo del motivo similar en la flauta): no se percibe entonces un diálogo, sino que cada uno de los interlocutores pareciera estar encerrado en sus propias ideas. La flauta entonces tiene intervenciones fragmentadas (divisio, distributio) a lo largo de toda la sección. Su primera aparición combina los motivos a1 y b, en la zona alta del registro, coincidiendo con el gesto enfático de la soprano aunque con sus propias "palabras". Recordemos que el re” fue la nota más alta que se alcanzó en el exordio con el motivo a, luego de una gradatio, que hacía referencia a la elevación de los aromas de la mirra como metáfora de la elevación del alma, en este caso, estamos por así decir "elevados en los cielos". La frase nos lleva a la mayor, tonalidad lejana afectivamente de si menor ${ }^{17}$, ya no patética, sino exaltada. La flauta interviene nuevamente luego de un compás de silencio con el motivo a’, que como recordamos aparecía en el exordio en la sección 2 caracterizada por los "sollozos". Pero aquí no encontramos el motivo plañidero y lo que sobresale es la idea de ascenso de a', sobretodo porque es repetido en gradatio ascendente luego de un compás de silencio. Luego de un nuevo compás de silencio la flauta retorna con tres intervenciones asiladas del motivo d', también en gradatio ascendente (cc. 54, 55 y 56). Constatamos que hasta ahora, los comentarios de la flauta tienden a ser enfáticos, con una exaltación cada vez mayor, lo que contrasta con la melancolía del exordio.

La armonía pasa por re mayor y llega a mi menor. Luego de otro compás de silencio interviene la flauta con su frase más larga conformada por el motivo e' (tresillos) y una gradatio en catábasis que desemboca en la dominante de si menor (podemos decir que la flauta termina con la figura de interrogatio), un compás antes de que la soprano cierre la sección con su frase cadenciando a si menor. El contenido descendente de la frase, la alusión al tópico de los "sollozos" en los tresillos, la figura de dubitatio que caracteriza al motivo d'(al igual que al motivo d) y el final de frase en interrogatio, nos acercan progresivamente al afecto y tonalidad principal de la pieza. Luego de la cadencia, el exordio se repite en su totalidad con función de confirmatio y peroratio a la vez, antes de abordar la parte B del aria.

Por lo tanto, podemos constatar que la refutación se realizó a través de los aspectos que enumeramos más arriba deducidos de las fuentes: disonancias (no especialmente en forma de retardos, aunque los hay, sino fundamentalmente
17. Si menor para Mattheson es "extraño, hosco y melancólico; es por lo que aparece tan raramente. Quizás es también la razón de que los Antiguos lo hubieran desterrado de sus conventos."; para Rameau es "dulce, tierno" y para Schubart expresa "paciencia, en espera tranquila de su suerte y de la resignación a la voluntad de Dios. Su lamento es tan dulce que nunca se desata en murmullos o chillidos injuriosos." Por el contrario, los mismos autores opinan sobre $\underline{\text { la }}$ mayor: "Esta tonalidad debe sobrecoger. Brilla inmediatamente, $y$ más para las pasiones plañideras y tristes que para la diversión.”; "alegría, júbilo"; "Este tono contiene declaraciones de amor inocente, satisfecho de su condición, [...] conviene particularmente al violín. Ver de nuevo al ser amado. alegría juvenil. Confianza, Dios.” Johann Mattheson, Das Neu-eröfnete Orchestre, Hamburgo,1713; JeanPhilippe Rameau, Tratado de armonía, París, 1722; Christian Friedrich Schubart, Ideen zu einer Ästhetik der Tonkunst, Viena,1806.(STEBLIN, 1981). 
melódicos a través de saltiduriusculi en la interválica de los motivos);modulaciones; "pasajes extrańos" o contrastantes; fragmentación (divisio) y elaboración motívica, junto con afectos que divergen o contrastan con el afecto principal.

En relación con este último punto, recordemos que Forkel asigna a la divisio la función de individualizar sentimientos generales. La fragmentación de la participación de la flauta en esta sección pareciera apuntar más claramente a la personalización del instrumento como un personaje del tejido dramático, ya que sus comentarios no hacen coincidir sus "palabras" con el texto musical de la soprano. Su "exaltación" se hace desde la indignación o la angustia, como afectos más individualizados y episódicos, que la depresión o el estupor predominante en el exordio, producto en las mujeres del hecho de encontrar la tumba vacía.
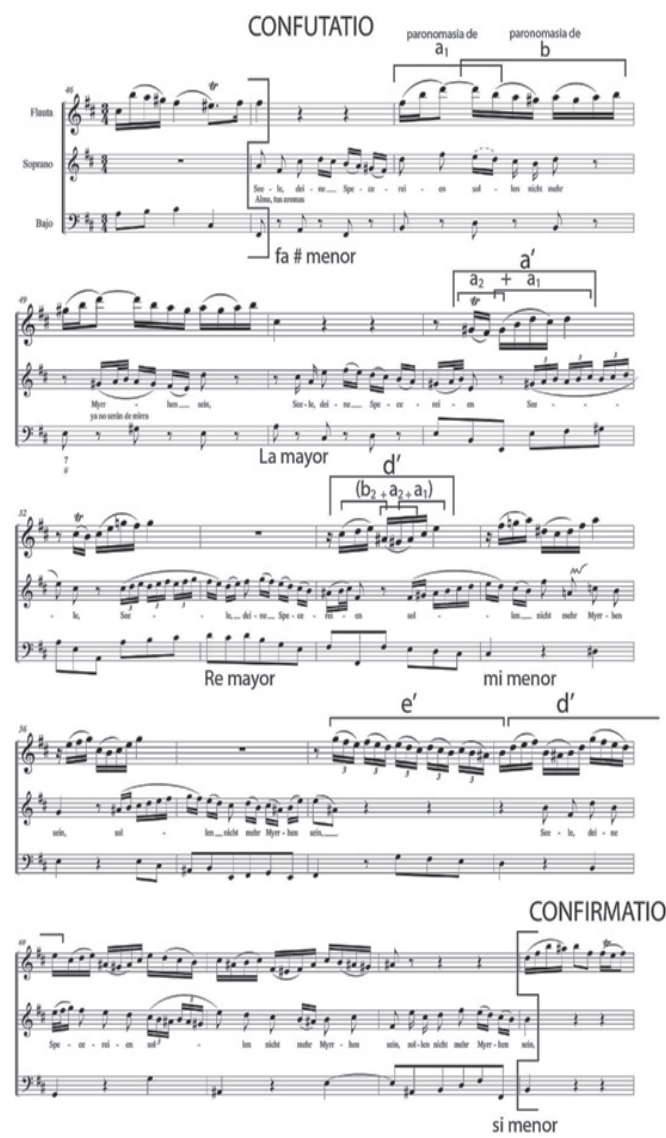

Ejemplo 2: Sección de confutatio del Aria Seele, Oratorio de Pascua, BWV 249 de Johann Sebastian Bach 
La parte B presenta aspectos refutatorios más complejos de los cuales sólo señalaremos la construcción de un nuevo motivo a partir de la hipotiposis de la corona de laurel. La corona de laurel hace referencia a la que se sostenía por sobre la cabeza de los generales romanos victoriosos en la ceremonia del triunfo; un esclavo se encargaba de hacerlo y cada tanto tenía la obligación de decirle al victorioso "recuerda que morirás" (memento mori) para que la gloria no lo ensoberbeciera. El texto poético del Aria dice que "sólo la corona de laurel", como metonimia del triunfo, "aliviará la angustia del alma": se refiere al triunfo sobre la muerte de la resurrección de Jesús y a la promesa de redención y vida eterna que esto implica.

Este texto aparece señalado con el único motivo del aria en que la flauta y la voz proceden paralelamente en noema, con intervalos de $6^{\text {ta }}$. Esto nunca había sucedido, salvo de forma fragmentaria en otros segmentos pero correspondiendo con motivos diferentes en cada una de las voces. Reproducimos en el ejemplo 3 los cc. 81-82. El motivo se repite de la misma manera en los cc. 85-86, 96-97 y 105-106.

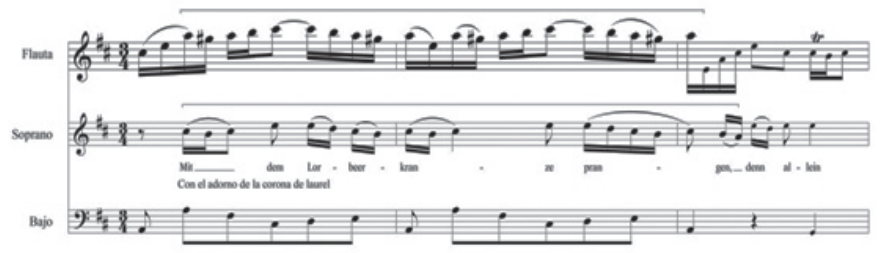

Ejemplo 3: Extracto de la parte B del Aria Seele, Oratorio de Pascua, BWV 249 de Johann Sebastian Bach

\section{CONSIDERACIONES FINALES}

Quizás la referencia a aspectos que la crítica tradicional suele denominar de "extra-musicales", como podrían ser la asociación de los motivos con determinados afectos o contenidos narrativos, puedan ser criticados. Es evidente que no abogamos por un tipo de escritura crítica similar a la favorecida por el Romanticismo que metaforizaba poéticamente el discurso sobre la música, la cual, por definición estética, era inefable. Es indudable también que asignar contenidos conceptuales o metafóricos (el ascenso del alma o de los vapores 
de la mirra) a estructuras sonoras es arbitrario. También es dificultoso, hoy día, casi trescientos años después, establecer los nexos entre significado discursivo y significante sonoro. Es probable que el código establecido en la época estuviera en condiciones dejar en claro este significado a un potencial receptor. Hay algunas estructuras más comentadas (como los descensos por grados y a veces cromáticos para los "sollozos") y otras menos documentadas, por lo cual hay que ser cauteloso en las asociaciones.

Sin embargo, el abordaje retórico de la música desde la metáfora del lenguaje en el período estudiado, daba por sentado que la ciencia musical "avanzaba" a favor de una inteligibilidad articulada. Aquí sólo resta señalar que el discurso entendido de esta manera es una herramienta fundamental para establecer criterios de interpretación. Saber que el instrumento puede representar un determinado "personaje" de un tejido dramático, o entender cómo funciona cada motivo en la confutación: tendiendo a la exaltación para luego volver al afecto plañidero en los tresillos (que por el contrario no están allí para mostrar un alarde de virtuosismo), llevará a adoptar estrategias prosódicas adecuadas en el momento de la ejecución. Consideramos relevantes para una eficiente práctica de esta música conocer estos recursos. El especial conocimiento de la forma que supone la dispositio retórica, puede orientar e inspirar al intérprete.

\section{REFERÊNCIAS BIBLIOGRÁFICAS:}

ARISTOTELES. El arte de la retórica. Buenos Aires: Eudeba, 2010.

BARTEL, Dietrich. MusicaPoetica. Musical-Rhetorical Figures in German Baroque Music. Lincoln y Londres: Universityof Nebraska Press, 1997.

Rhetoric in German Baroque Music: Ethical Gestures. The Musical Times, 144(1885), 15, 2007. Disponible en: http://doi.org/10.2307/3650721. Consultado en 02 mai. 2017.

BARTHES, Roland. Investigaciones retóricas I. La antigua retórica. Buenos Aires: Tiempo contemporáneo, 1974 
BERISTÁIN, Helena. Diccionario de Retórica y Poética. México DF: Editorial Porrúa, S. A, 1995.

BONDS, Marc Evan. Wordless Rhetoric. Musical Form and the Metaphor of the Oration. Cambridge y Londres: Harvard University Press, 1991.

La música como pensamiento : el público y la música instrumental en la época de Beethoven. Acantilado, 2014. Disponible en http://www.acantilado.es/catalogo/lamusica-como-pensamiento/. Consultadoenen 3 mai. 2017.

BRAUSCHWEIG, K. (2001). Genealogy and MusicaPoetica. In: Seventeenth- and Eighteenth-Century Theory. Acta Musicologica, 73(1), 45, 2001. Disponible enhttp://doi. org/10.2307/932809. Consultado en 04 mai. 2017.

BROSSARD, Sébastien. de. Dictionnaire de musique. Paris: Christophe Ballard, 1705.

BUELOW, Georg. Teaching Seventeenth-Century Concepts of Musical Form and Expression: An Aspect of Baroque Music. In: College Music Symposium, 27, 1987, p. 1-13.

BUELOW, Georg. Music, rhetoric, and the concept of the affections: a selective bibliography. George J. Buelow Source: Notes, v. 30, n. 2, 1970, p. 250-259. Disponible en http:// www.jstor.org/stable/895972. Consultadoen 2 mai. 2017.

Rhetoric and Music. In: SADIE, Stanley (ed.). The New Grove Dictionary of Music \& Musicians. London: Macmillan, 2000, p. 793-803.

Mattheson, Johann. In Grove Music Online (Vol. 1). Oxford: Oxford University Press. Disponible enhttp://doi.org/10.1093/gmo/9781561592630.article.18097. Consultadoenen 2 mai. 2017.

BUKOFZER, Manfred. Allegory in Baroque Music. In: Source Journal of the Warburg and Courtauld Institutes, v. 3, n. 12, 1939, p. 1-21. Disponible enhttp://www.jstor.org/ stable/750188. Consultadoenen 3 mai. 2017. 
BURMEISTER, Joachim.Musica Poetica. Kassel [Rostock]: Bärenreiter[Myliander], 1954 [1606].

BUTLER, GregoryG.. Fugue and Rhetoric. Journal of Music Theory, v. 21, n.1, 1971, p. 49-79. Disponible enhttp://doi. org/10.2307/843479. Consultadoenen 2 mai. 2017.

BUT', John. Bach Interpretation / articulation Marks in Primary Sources ofJ.S. Bach. Cambridge: Cambridge University Press, 1990.

Playing with History. The Historical Approach to Musical Performance. Cambridge, New York: Cambridge UniversityPress, 2002.

Bach's dialogue with modernity: perspectives on the passions. Cambridge University Press, 2010. Disponible en http://www.cambridge.org/gb/academic/subjects/music/ eighteenth-century-music/bachs-dialogue-modernity-perspectives-passions? format $=$ PB\&isbn $=9781107404601 \# \mathrm{ksEB}$ pIxlgleiXu4t.97. Consultadoenen 2 mai. 2017.

CICERO, MarcoTullius, \&Iso, José Javier. Sobre el orador. Gredos Editorial, 2002. Disponible en http://www.editorialgredos.com/biblioteca_clasica_gredos/_18a_sobre_orador. Consultado enen 2 mai. 2017.

CICERÓN, M. T., \&Núñez, Sebastián. (1997). La invenciónretórica. Gredos, 1997. Disponible en http://www. editorialgredos.com/biblioteca_clasica_gredos/_188_invencion_retorica. Consultado enen 2 mai. 2017.

CIRILLO, A. (2016). Universidad de Murcia - Johann Joachim Quantz y su aportación a la cultura musical del siglo XVIII. Universidad de Murcia, 2016. Disponible en https://plu.mx/ um/a/?repo_url=http://hdl.handle.net/10201/47863. Consultado enen 2 mai 2017.

CIVRA, Ferrucio. MusicaPoetica, Introduzionealla retorica musicale. Torino: UTET Librería, 1991. 
CLERC, Pierre-Alain. Discours sur la RhetoriqueMusicale. et plus particulierement la Rhetoriqueallemande entre 1600 et 1750. Peyresq: por el autor, 2000. Disponible enwww.cdlhem. ch/hemClassique/corpsEnseignant/index.php?pageIdUrl=83. Consultado enen 3 mai. 2017.

COBLEY, Paul. Communication and Verosimilitude in Eigthteen-Century. In MIRKA, Danuta\&Agawu, Kofi (eds.), Communication in Eigthteen-Century Music. Cambridge: Cambridge UniversityPress, 2008, p. 13-33.

CRAVERI, Benedetta.La cultura de la conversación. Mexico: Fondo de Cultura Económica, 2004. Retrieved from https:// elfondoenlinea.com/Detalle.aspx?ctit=003537R

DESCARTES, René. Renati Descartes Musicae compendium. Utrecht: Johann Jansson, 1650.

DESCARTES, René;Gascon, Onaindia; Izquierdo, Augustín. Las pasiones del alma. Madrid: Edaf. 2005

DREYFUS, Laurence. Early Music Defended against its Devotees: A Theory of Historical Performance in the Twentieth Century. In: The Musical Quarterly, v. 49, n. 3, 1983, p. 297-322. Disponible en: http://doi.org/10.1093/mq/ LXIX.3.297. Consultadoenen 2 mai. 2017.

FADER, Don. The Honnête homme as Music Critic_Taste, Rhetoric, and Politesse in the 17th Century. In: The Journal of Musicology, v. 20, n. 1, 2003, p. 3-44. Disponible en: http:// doi.org/10.1525/rep.2014.126.1.1.1. Consultadoenen 3 mai. 2017.

FORKEL, Johann Nikolaus. Über die theorie der Musik, insofernsieLiebhabern und KennernNotwending und nützlichist. Göttingen: Vandenhoek, 1777.

GEMINIANI, Francesco. A Treatise of Good Taste in the Art of Musick. Londres: [por el autor], 1749. 
GEMINIANI, Francesco. The Art of Playing the Violin, Opera $I X$. Londres: [por el autor], 1751.

GJERDINGEN, Robert. O..Music in the Galant Style. Oxford: Oxford University Press, 2007.

GRESSET, Jean-Baptiste.-Louis.Discours sur l'harmonie. Paris: J. N. Le Clerc, 1737.Disponible en: http://bibliotheque.bordeaux.fr/in/faces/details.xhtml?id=h::BordeauxS_ PF15678_2Res\&jscheck=1. Consultadoenen 2 mai. 2017.

HAYNES, Bruce. The End of Early Music. A Period Performer's History of Music for the Twenty-First Century. Oxford y Nueva York: Oxford University Press, 2007.

HEINICHEN, Johann David. Der General-Bass in der Composition. Dresden: [por el autor] 1728.

HOTTETERRE, J. (1719). L'art de préluder sur la flûtetraversière, sur la flùte à bec, sur lehautbois et autresinstrumens de dessus. Op.7.Firenze [Paris]: SPES[Focault], 1719. Paris (ed. M. Castellani).

HUME, David. De la tragedia y otros ensayos sobre el gusto. Buenos Aires: Biblos, 2003 (traducción M. Marey).

KIRKENDALE, Ursula. The Source for Bach'sMusical Offerin.: TheInstitutiooratoria of Quintilian. In: Journal of the American Musicological Society, v. 33. n.1, 1980, p. 88-141. Disponible enhttp://doi.org/10.2307/831204. Consultadoenen 3 mai. 2018.

KIRNBERGER, Johann Philipp. Die Kunst des reinenSatzes in der Musik. Berlin und Köngisberger: Decker und Hartung, 1774.

KLIBANSKY, Raymond, PANOFSKY, Erwin, \& SAXL, Fritz. Saturno y la melancolía / Estudios de historia de la filosofía de la naturaleza, la religión y el arte. Madrid: Alianza editorial, 1991.

LA BORDE, Jean-Benjamin de. Essai sur la musique ancienne et moderne. Paris: Pierres, 1780. 
LACÉPÈDE, Étienne de. La poétique de la musique . Paris: L'Imprimerie de Monsieur, 1785. Disponible en: http://gallica.bnf.fr/ark:/12148/bpt6k114571v.r=La poétique de la musique par M. le Cte. de Lacépède?rk=107296;4. Consultado enen 3 mai. 2017.

LENNEBERG, Hans. Johann Mattheson on Affect and Rhetoric in Music (II). Journal of Music Theory, v. 2,n. 2, 1958, p. 193. http://doi.org/10.2307/843199. Consultadoenen 2 mai. 2017.

Johann Mattheson on Affect and Rhetoric in Music (I). Journal of Music Theory, v. 2, n. 1, 47. Disponible en: http://doi.org/10.2307/842930. Consultadoenen 2 mai. 2017.

LÓPEZ CANO, Rubén. La ineludible preeminencia del gozo: el Tratado de las pasiones del alma de René Descartes en la música de los siglos XVII y XVIII. In:Simposio "Descartes Vivo." Universidad Autónoma de Querétaro, 2016. Disponible en: www.lopezcano.net. Consultado enen 2 mai. 2017.

LÓPEZ CANO, Rubén. Arsmusicandi: la posibilidad de una retórica musical desde una perspectiva intersemiótica. In:Congreso "El horizonte interdisciplinario de la retórica." Instituto de Investigaciones Filológicas (UNAM), 1998. Disponible en: www.lopezcano.net. Consultado enen 2 mai. 2017.

LÓPEZ CANO, Rubén. Música y retórica en el barroco. Barcelona: Amalgama textos, 2011.

MARPURG, Friedrich Wilhelm. Anfangsgründe der theoretischenMusik. Leipzig: Breitkopf, 1757.

MATHER, Betty Bang. Interpretation of french music from 1675-1775 for woodwind and other performers.Mcginnis\& Marx Music Pub., 1989.

MATTHESON, Johann. Das neu eröffneteOrchestre. Hamburg: Schiller, 1713. 
MATTHESON, Johann. Der brauchbare Virtuoso. Hamburg: Schiller- und Kißner, 1720. Disponible en: http://imslp.org/ wiki/Der_brauchbare_Virtuoso_(Mattheson,_Johann). Consultadoenen 1 mai. 2017.

MATTHESON, Johann. Der vollkommene Capellmeister. Hamburgo: Cristian Herold, 1739.Disponible en: http:// ks.petruccimusiclibrary.org/files/imglnks/usimg/7/7b/ IMSLP67752-PMLP136831-Mattheson,_Der_vollkommene_Capellmeister.pdf. Consultadoenen 1 mai. 2017.

MONTECLAIR, MichelPignolet. de. Principes de musiquedivisezenquatre parties, Paris: [por el autor], 1736.

NASARRE, Pablo..Escuela música según la práctica moderna. Zaragoza: Diego de Larumbe, 1724.

NEUMANN, Friedrich. Ornamentation in Baroque and PostBaroque music. Princeton: Princeton UniversityPress, 1978.

NÚÑEZ, Sebastián, CICERO, M. T., \& CORNIFICIUS, rhetor.Retórica a Herenio. Madrid: Gredos, 1997. Disponibleen:http://www.editorialgredos.com/biblioteca clasica_gredos/_21f_retorica_herenio.Consultado en em 1 mai. 2017.

PALACIOS QUIROZ, Rafael. La pronuntiatiomusicale: une interprétationrhétoriqueauservice de Händel, Montéclair, C. P. E. Bach et Telemann. Paris: UniversitéParisSorbonne (tesis doctoral), 2012.

PÉRSICO, Gabriel. Música, retórica y comunicación: esbozo de análisis retórico de una pieza para flauta travesera de George Philipp Telemann. In:Revista Del Instituto de Investigación Musicológica "Carlos Vega". UCA, v. 23, 2009, p. 275-313. Retrieved from http://bibliotecadigital.uca.edu.ar/repositorio/revistas/retorica-musica-barroca-hermeneutica-persico. pdf. Consultadoenen 1 mai. 2017.

PETERMAN, Lewis Emanuel. Michel Blavet's Breathing Marks: A Rare Source for Musical Phrasing in Eighteenth- 
Century France.Performance Practice Review, v. 4, n.2, 1991,p. 186-198. Disponible en: http://doi.org/10.5642/ perfpr.199104.02.04. Consultadoenen 1 mai. 2017.

QUANTZ, Johann Joachim.VersucheinerAnweisung die FlötetraversierezuspielenBerlin: Voss, 1752. Disponible en: http://imslp.org/wiki/Versuch_einer_Anweisung_die_Flöte_ traversiere_zu_spielen_(Quantz,_Johann_Joachim). Consultadoenen 1 mai. 2017.

RODRÍGUEZ, H. ArsRethorica. In Centro de Estudios de Música Antigua, UCA. San Vicente: por el autor, 1996.

ROUSSEAU, Jean-Jacques. (1768). Dictionnaire de $M u$ sique. ParisChez la veuve Duchesne, 1768. Disponible en: http://archive.org/stream/dictionnairedem00rous\#page/30/ mode/2up. Consultadoenen 1 mai. 2017.

SAINT-LAMBERT, Michel de Les principes du clavecin. Paris: Ballard, 1702.

SCHULENBERG, David. "Musical Allegory" Reconsidered: Representation and Imagination in the Baroque. TheJournalofMusicology, n. 3, v. 2,1995, p. 203-239. Disponible en: http://doi.org/10.2307/764105. Consultado enen 2 mai 2017.

TARLING, Judy. The weapons of Rhetoric / a guide for musicians and audiencies. Hertfordshire: Corda Music Publications, 1994.

VAN EYGHEN, Peter. Rhetorics and Affections in Music ca. 1600 - ca. 1750. La Haya: Royal Conservatory The Hague, 2001.

VEILHAN, Jean-Claude. Les regles de l'interpretationmusicale a l'epoqueBaroque: (xviie-xviiie s.), generales a tous les instruments. A. Leduc, 1977. 
VICKERS, Brian. Figures of rhetoric/Figures of music? Rhetorica, v. 1, n. 2, 1984, p. 1-44. Disponible em: http://doi. org/10.1525/rh.1984.2.1.1. Consultado en em 1 mai. 2017.

VIEVILLE, Jean-Louis le Cerf de laComparaison de la musiqueitalienne et de françoise. Bruxelles: Foppens, 1705.

WAISMAN, Leonardo. Música Antigua y autenticidad: ideología y práctica. In Décimas Jornadas Argentinas de Musicología. Buenos Aires: AAM, 1995.

WEBER, William. La gran transformación en el gusto musical: la programación de conciertos de Haydn a Brahms. Buenos Aires: Fondo de Cultura Económica, 2011. Disponivle en: https://elfondoenlinea.com/Detalle.aspx?ctit=003663R. Consultado enen 1 mai. 2017. 